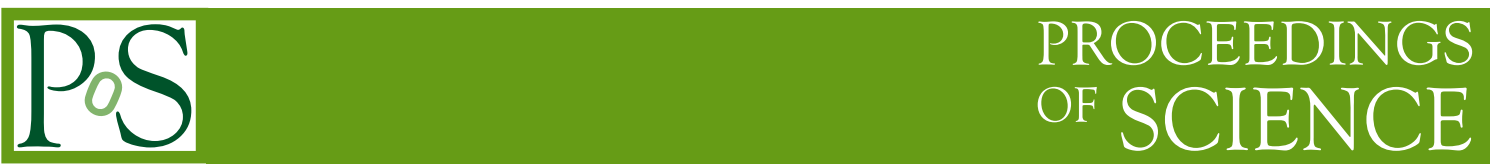

\title{
LOFT Simulation Toolkit
}

\author{
Christian Schmid*, Thorsten Brand, Matthias Kühnel, Michael Wille, Jörn Wilms \\ Dr. Remeis-Observatory \& ECAP, University Erlangen-Nuremberg, Germany \\ E-mail: christian.schmidesternwarte.uni-erlangen.de
}

Riccardo Campana, Immacolata Donnarumma, Yuri Evangelista

INAF-IAPS Roma, Italy

The Large Observatory For X-ray Timing (LOFT) has been selected by ESA as a candidate for the Cosmic Vision M3 launch slot. The mission concept comprises a Large Area Detector (LAD), which is a collimated instrument with an assembly of Silicon Drift Detectors (SDDs) providing an effective area of $\sim 10 \mathrm{~m}^{2}$ at $8 \mathrm{keV}$ and excellent timing capabilities. The second instrument aboard LOFT, the Wide Field Monitor (WFM), consists of several coded mask cameras monitoring a large fraction of the sky and therefore providing the capability to detect transient events.

We have developed a Monte Carlo simulation software in order to investigate the performance of LOFT during the current assessment phase. The simulation produces a sample of virtual photons for a given X-ray source specification, which is defined in the SIMPUT file format. These photons can be processed by a model of the LAD contained in the software package or with a particular instrument simulation of the WFM developed at INAF/IAPS Roma. For access to simulations of the LAD a web interface is provided.

In this paper we present the general design of the simulation software and show an analysis of the timing performance of the $\mathrm{LAD}$ as an example illustrating its capabilities.

"An INTEGRAL view of the high-energy sky (the first 10 years)" 9th INTEGRAL Workshop and celebration of the 10th anniversary of the launch,

October 15-19, 2012

Bibliotheque Nationale de France, Paris, France

\footnotetext{
* Speaker.
} 


\section{Introduction}

The LOFT mission $[2,7]$ will be a very powerful tool to address physical questions related to strong-field gravity and ultra-dense matter. Its LAD with an energy range of $2-80 \mathrm{keV}$ (the range $30-80 \mathrm{keV}$ has a coarse energy resolution) provides a gigantic effective area of $\sim 10 \mathrm{~m}^{2}$ at $8 \mathrm{keV}$ and an excellent time resolution [11]. The WFM will enable the monitoring and the detection of transient X-ray sources in the same energy range [3].

LOFT has been selected by ESA as a candidate for the Cosmic Vision M3 launch slot in the time frame 2022-2024 and is currently undergoing an assessment phase. In this context numerous investigations of the technical and scientific aspects of both instruments are required. Many of these studies are based on simulations in order to account for a large variety of relevant instrumentspecific features.

In the following we present a Monte Carlo simulation toolkit that allows a detailed analysis of the performance of the instruments.

\section{Simulation Software}

The simulation software has originally been developed for performance investigations of a number of other astronomical X-ray instruments, such as eROSITA on SRG and IXO/Athena [10]. It has been adapted to the setup of the LOFT mission.

The software is written in $\mathrm{C}$ and implements a common interface by using the APE/PIL library for parameter input. Access to input and output files is based on the FITS file format in order to provide compatibility with common data analysis tools. This approach is also supported by using standard calibration files to define the properties of the simulated instruments. The setup of a particular instrument model is defined by a set of parameters in an XML configuration file. The software therefore provides flexibility to investigate the impact of modifications in the instrument design as well as physical accuracy for a detailed performance analysis.

The simulation is processing individual photons. A simulated observation of a particular target comprises two steps. First a sample of X-ray photons is produced based on a given source specification. Then these photons are input to the respective instrument model. In the implemented approach a simulation is set up as a pipeline of several tasks, as shown in Fig. 1.

The first part of the pipeline, which produces a sample of X-ray photons, can deliver input for the LAD as well as for the WFM instrument model. The presented software package contains a model for the LAD comprising the collimator and the array of SDDs. A model for the WFM is developed separately $[5,6]$. It accepts the sample of generated X-ray photons as input a simulates an observation with the coded mask cameras.

\subsection{Photon Sample}

In order to produce a sample of photons, it is necessary to specify the observed X-ray sources. The simulation software relies on the SIMPUT file format [9] ${ }^{1}$. It provides a powerful approach to describe astronomical sources with various features comprising detailed energy spectra, timing

\footnotetext{
${ }^{1}$ http: //hea-www . harvard.edu/HEASARC/formats/simput-1.0.0.pdf
} 


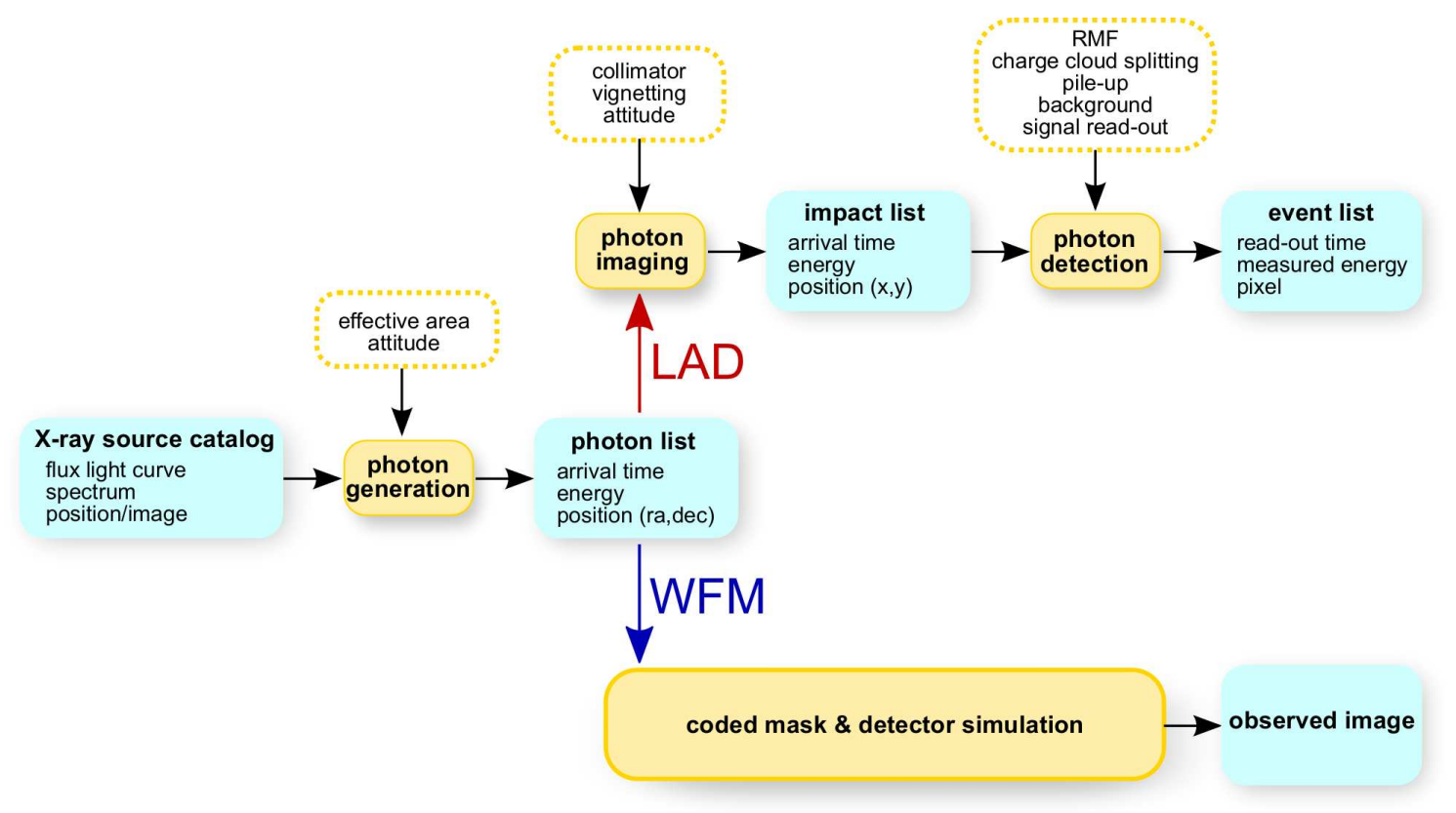

Figure 1: A simulation is set up as a pipeline of several tasks. The photon generation as a universal tool can deliver input for both the LAD and the WFM instrument models.

properties, and spatial extension as input for simulations. The source specifications in SIMPUT are independent of the regarded instrument. We provide a sample of SIMPUT files assembled for different source categories, which can be used as input for simulations.

The individual photons produced from the source specification are characterized by their energy, their arrival time at the instrument, and their direction of origin.

\subsection{LAD Instrument Model}

The produced photons can be processed with both instrument models of the LAD, as presented below, and the WFM, which is described by $[5,6]$. The LAD consists of 6 panels with 21 modules each. One module comprises 16 SDDs. The SDDs are illuminated from the front through a collimator limiting the field of view and avoiding photons from sources other than the target.

Instead of taking into account the physical interaction processes of individual photons with the collimator or the detector material as in a GEANT4 $[1,8]$ simulation, our software relies on a simplified approach in order avoid extensive computational effort. The relevant steps are modeled by randomization processes based on standard calibration files such as the ARF and the RMF, reflecting the instrument characteristics related to the collimator and the detector and guaranteeing physical accuracy. The transmission of photons through the collimator and the determination of the their impact positions of the SDDs depends on the pointing direction of the instrument, which is specified in a particular attitude file, and the diameter of the field of view. Additional events are inserted according to the estimated LAD background model. The spread of the charge cloud produced by an absorbed photon and the measured signal are calculated with the model presented by [4]. The implementation of the signal processing focuses on the relevant features in order to analyze phenomena such as dead time effects or pile-up. 


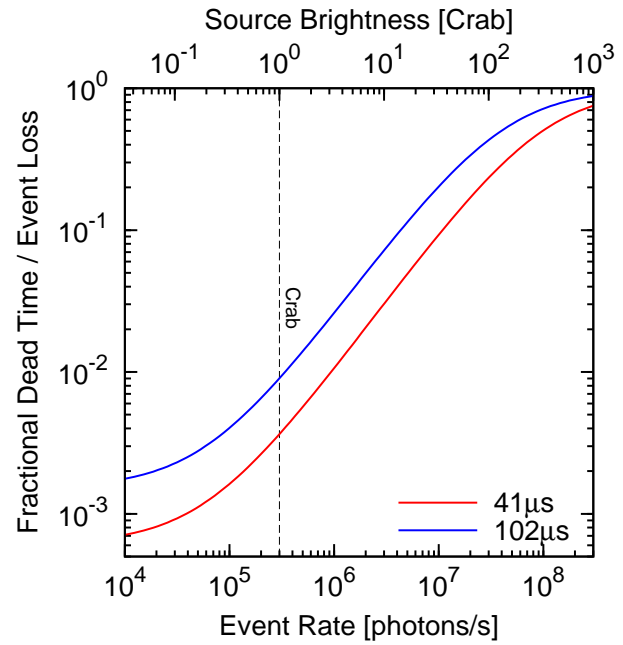

(a) Attenuation of the count rate observed with the LAD due to dead time introduced by the analog to digital conversion of the detected signals. Data are shown for different clock frequencies. At a flux of $1 \mathrm{Crab}$ the losses are well below $1 \%$.

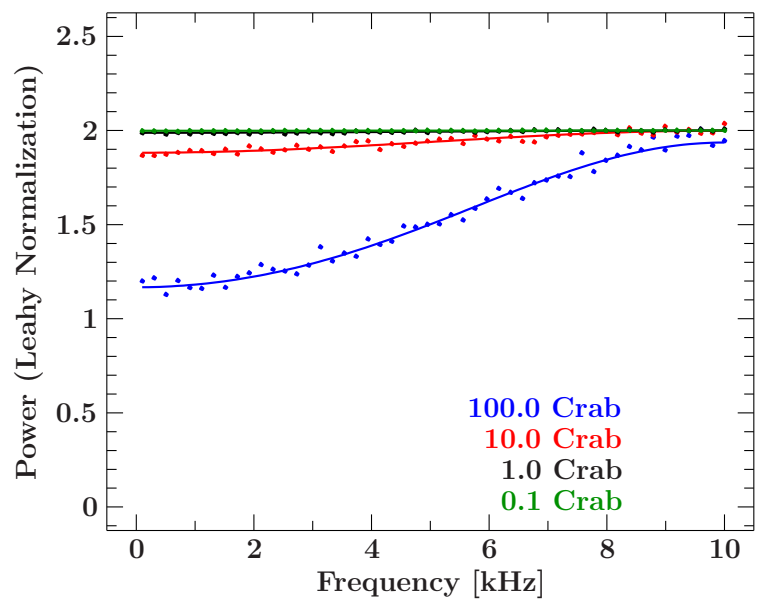

(b) Power spectrum of Poisson noise for different source fluxes, as observed with the LAD. At very high fluxes the shape significantly deviates from the theoretical value of 2 (in the selected Leahy normalization) due to the influence of the dead time. The dots represent simulated data and the straight line an analytical model [12].

Figure 2: Dead time effects on observations with the LAD.

The primary output of the simulation is an event file with the individual detected photon events. These data can be further processed to spectra and light curves with the corresponding auxiliary tools contained in the software package or with common data analysis tools.

\section{LAD Dead Time Analysis}

Since a major part of the scientific goals of the LAD relies on highly resolved measurements in the time domain, a detailed knowledge of the respective instrument properties is essential in order to assure the correct interpretation of the measured data and the possibility to distinguish between instrumental and source-intrinsic features. The presented simulation software is suitable for elaborate technical investigations of the relevant features.

An important aspect is the dead time introduced by the signal processing by the front-end electronics. The conversion of the detected charge signals to a digital form requires a certain interval of $41 \mu \mathrm{s}$ or $102 \mu \mathrm{s}$, depending on the implemented clock. During this period the respective part of the detector is insensitive to any further incident photons such that they are discarded and therefore missing in the measured data. As the duration of this interval is not extended by the discarded photons, the dead time behavior of the LAD is characterized as non-paralyzable [12].

The effect of this dead time attenuation is especially relevant for observations of very bright sources. In this regime the detected event rate can be significantly lower than the actual incident rate. However, our analysis shows that the fractional dead time affecting the LAD is less than $1 \%$ for a source flux of $1 \mathrm{Crab}$, as displayed in Fig. 2(a).

In addition to the mere event loss, dead time can cause artifacts affecting the timing analysis of the observed data. Events following directly after a previous event at the same part of the detector 
are discarded due to the insensitivity of the device. This effects results in a suppression of short intervals in between subsequent events.

In order to illustrate this phenomenon, the plot in Fig. 2(b) displays a power spectrum for sources with constant brightness and purely Poisson-determined photon number fluctuations at different fluxes. In the selected Leahy normalization the power spectra therefore ideally should have a constant value of 2 . For fluxes up to several Crab the data are not affected by any significant dead time effects. Whereas for extremely bright sources the observed power spectrum exhibits particular features visible as deviations from the pure Poisson noise shape. These effects have to be taken into account for data analysis in order to be able to distinguish between instrumental and source-intrinsic phenomena. Therefore a detailed understanding of all contributions is required, which can be obtained from simulations.

The graph in Fig. 2(b) contains simulated data as well as an analytical dead time model according to [12]. In contrast to the analytical model, the simulations enable the analysis of more sophisticated timing effects beyond the dead time introduced by the analog to digital conversion of the detected signals. Examples therefore are pile-up of multiple photons, charge cloud splitting, or a more elaborate treatment of the signal processing chain.

\section{Web Interface for LAD Simulations}

For fast and easy access to the simulation software, a web interface is provided on the official LOFT web site ${ }^{2}$ and on a web server at ECAP ${ }^{3}$. In Fig. 3 a screenshot of this interface is displayed. It allows the setup of an observation scenario with a particular set of predefined parameters. The modification of the instrument configuration, which is set to the default baseline, is not possible.

There are two different ways to define the observed X-ray source via the interface. Either a model of a single point-like source can be assembled from various spectral components such as a power law or a black body spectrum, and with optional time variability defined by several components of a power spectrum. Or a SIMPUT file with one or multiple source definitions can be uploaded to the server.

Apart from the simulated exposure time, the user can select among various data products to be delivered by the simulation. Possible options are an event file containing the individual detected photon events, an energy spectrum as a histogram of the energies of the detected events, a light curve with a selectable time resolution, and a SIMPUT file describing the observed target for reproducibility of the simulation setup. The simulation is initiated by a click on the respective button. After its completion the selected data products can be downloaded and analyzed.

\section{Conclusion}

The presented simulation software is a powerful tool to investigate the technical and scientific performance of the LOFT instruments. As shown with regard to the dead time of the LAD, it is possible to analyze the impact of timing effects on the measured data. This is essential to understand the properties of the instrument.

\footnotetext{
${ }^{2}$ http://www.isdc.unige.ch/loft/index.php

${ }^{3}$ http://cetus.sternwarte.uni-erlangen.de/simloftsim/
} 


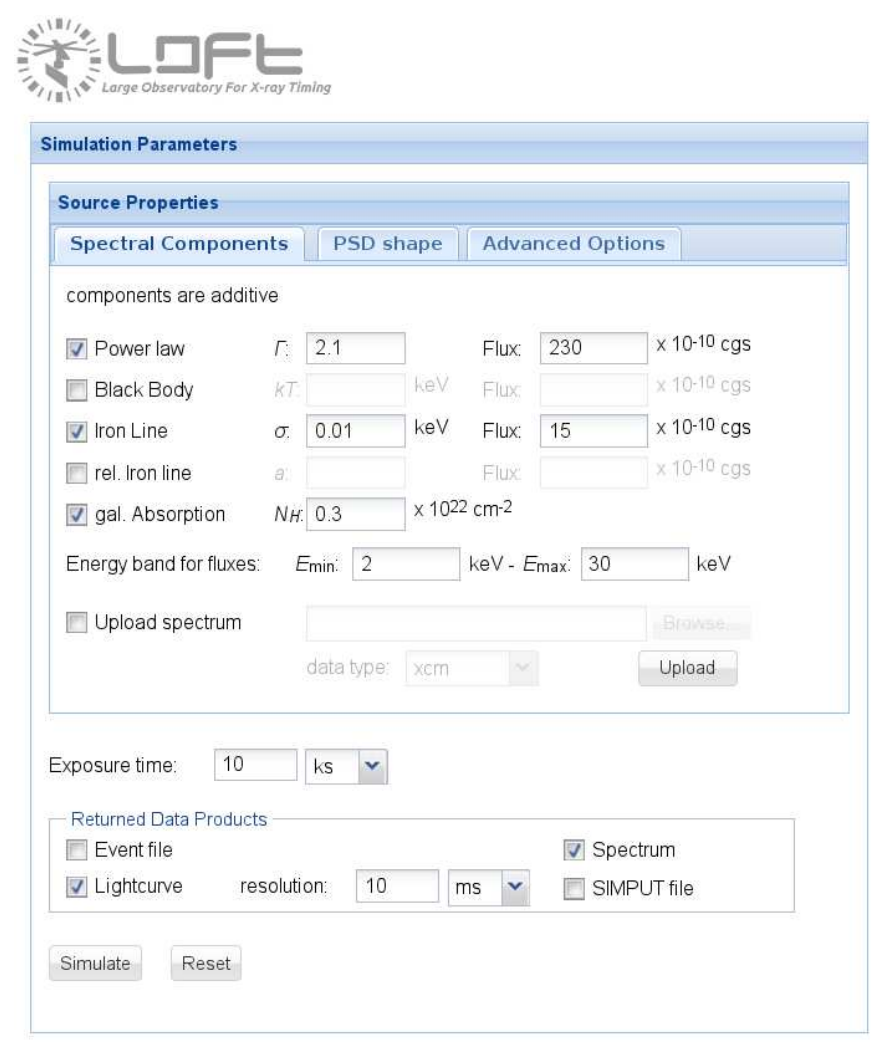

Figure 3: Web interface for simulations of the LAD.

\section{Acknowledgments}

This work has been supported in part by the Bundesministerium für Wirtschaft und Technologie under Deutsches Zentrum für Luft- und Raumfahrt grant number 50001111.

\section{References}

[1] Allison, J., et al., 2006, ITNS, 53, 270

[2] Bozzo, E., et al., 2012, this volume

[3] Brandt, S., et al., 2012, Proc. of SPIE, 8443, 2 G

[4] Campana, R., et al., 2011, NIMPA, 633, 22

[5] Donnarumma, I., et al., 2012, Proc. of SPIE, 8443, 5Q

[6] Evangelista, Y., et al., 2012, Proc. of SPIE, 8443, 5P

[7] Feroci, M., et al., 2012, Experimental Astronomy, 34, 415

[8] Geant4 Collaboration, et al., 2003, NIMPA, 506, 250

[9] Schmid, C., et al., 2011, tech. report

[10] Schmid, C., 2012, PhD thesis

[11] Zane, S., et al., 2012, Proc. of SPIE, 8443, 2F

[12] Zhang, W., et al., 1995, ApJ, 449, 930 